KENNEDY, R. (1986) Work of the day: aspects of work with families at the Cassel Hospital. In The Family as Inpatient (eds R. Kennedy, A. Heymans \& L. Tischler. London: Free Association Books.

KERNBERG, O. (1975) A systems approach to priority setting of interventions in groups. International Journal of Group Psychotherapy, 25, 251-275.

- (1980) Internal World and External Reality. New York: Jason Aronson.

- (1981) Some issues in the theory of hospital treatment. Tidsskr Nor Loegeforen, 101, 837-843.
LEVINE, H. B. (1980) Milieu biopsy: The place of the therapy group on the in-patient ward. International Journal of Group Psychotherapy, 30, 77-93.

MaIN, T. F. (1957) The ailment. British Journal of Medical Psychology, 30, 129-145.

MENZIES, I. E. P. (1959) The functioning of social systems as a defence against anxiety: a report on a study of the nursing service of a general hospital. Human Relations, 13, 95-121. Reprinted in Containing Anxiety in Institutions (I. E. P. Menzies Lyth) (1988). London: Free Association Books.

\title{
Services for pregnant drug users
}

\author{
M. London, Consultant Psychiatrist, Fulbourn Hospital, Cambridge CB1 3DF \\ (correspondence); R. CALDWELl, Senior Biochemist, Poisons Unit, \\ New Cross Hospital, Avonley Road, London SE14; and M. LIPSEDGE, \\ Consultant Psychiatrist, Department of Psychiatry, Guy's Hospital, London SE1
}

Drug and alcohol misuse during pregnancy poses a threat to the health of both the developing foetus and the mother yet this complex clinical problem often falls between different stools within the health services. A mother would first have to recognise herself as a drug or alcohol misuser needing help before she came within the ambit of the addiction services. On the other hand, the obstetric agencies, even when they identify a drug problem, usually do not regard themselves as having a role. General practitioners are most likely to have an overall view and therefore to be in the best position to manage the problem. However, many misusers do not register with GPs and, despite calls to the contrary, GPs have not become more widely involved in the management of addiction.

\section{The Project}

The Pregnant Misuser Project was started in Guy's Hospital in December 1987 with the aim of ascertaining the size of the problem and providing a service where one was lacking. Local services for misusers consist of a non-statutory shop-front agency and a drug dependency unit. In order to estimate the size of the problem, random urine surveys of pregnant mothers were carried out in the antenatal clinic. A midwife explained the purpose of the survey to mothers booking into the clinic for the first time. They were told that their urine would be tested for alcohol, tranquillisers or illicit substances but that whatever the result their antenatal care would continue regardless. The urine samples were screened for opiates and barbiturates by Thin Layer Chromatography (TLC) and for benzodiazepines, cannabis, cocaine and amphetamines using immuno assays. All mothers were later informed of the results of their tests. Such a survey obviously raised ethical issues but most of the staff felt that these issues were adequately addressed by conducting the survey on a voluntary, open basis.

Once a week one of the authors (MLn) was available to see individual mothers in the antenatal clinic. This would be arranged by the midwives. They would 
TABLE 1

Urine survey of 218 pregnant mothers

\begin{tabular}{c}
$\begin{array}{c}\text { Number of } \\
\text { mothers }\end{array}$ \\
\hline
\end{tabular}

Alcohol

Opiates: Methadone

Heroin metabolists

Benzodiazepine

Refused to provide a sample

No substance detected

TABLE II

Urine survey of 151 pregnant mothers

\begin{tabular}{lc}
\hline & $\begin{array}{c}\text { Number of } \\
\text { mothers }\end{array}$ \\
\hline Opiates: Dextropropoxyphene & 1 \\
& Heroin and cannabis \\
Methadone, cannabis & 1 \\
and benzodiazepines & 1 \\
Cocaine and cannabis & 2 \\
Benzodiazepine & 1 \\
Cannabis only & 6 \\
Refused to provide a sample & 4 \\
No substance detected & 135 \\
\hline
\end{tabular}

either identify themselves to the midwives at the time of booking into the clinic or they would be detected by way of the urine tests. At the interview their drug and alcohol use would be assessed and where appropriate treatment options discussed. This ranged from one or more counselling sessions in the antenatal clinic to referral to other treatment agencies. Opiate addicts could also receive a prescription for methadone as part of either maintenance treatment during pregnancy or detoxification within the antenatal clinic setting. This could be extended to their partners where appropriate. Confidentiality was maintained throughout and GPs informed only when prescribing occurred.

\section{The surveys}

Two surveys were carried out. The first tested 218 mothers for alcohol, opiates, benzodiazepines and amphetamines (Table I). Opiates were found in 1\% and alcohol in $2 \%$ of mothers. A total of $4 \%$ of the sample either showed alcohol or drugs in their urine or refused to provide a specimen.

In the second survey 151 pregnant mothers were additionally tested for cocaine and cannabis (Table II). Opiates were found in $2 \%$, cocaine in $1 \%$ and cannabis in $6.5 \%$ of mothers. Although tests for alcohol were performed, none were positive. Nevertheless, one in ten of the mothers surveyed either refused to provide a sample or were positive for drugs.

\section{The clients}

Beyond those detected by way of the urine test surveys, four additional mothers were identified as opiate users. This constituted a total of 12 opiate users identified during the first 12 months of the Pregnant Misuser Project. Three of these showed dextropropoxyphene in the urine but none came forward to be interviewed and assessed. Thus it is not known whether they were misusing analgesics for pleasure or taking these drugs therapeutically, however inappropriately. The fourth was identified only after delivery when her baby developed opiate withdrawal symptoms. During her addiction career she had received treatment from a drug dependency unit and spent six months in a residential treatment unit. The fifth and sixth mothers were already attending a shop-front agency and were drug-free at the start of the project. Both successfully delivered without neonatal withdrawal symptoms. One had received treatment from both a drug dependency unit and a residential unit, while the other had never engaged in treatment prior to her pregnancy. The seventh and eighth mothers were already in treatment receiving prescribed methadone when they became pregnant. One was being treated by her GP and the other by a drug dependency unit. The former delivered during the course of the project and, although she was being prescribed only $10 \mathrm{mg}$ methadone daily, she was probably abusing other opiates as her baby developed marked withdrawal symptoms.

The ninth mother presented late in pregnancy and was referred on to an in-patient treatment unit. She subsequently delivered without any neonatal withdrawal symptoms. She had no previous history of treatment other than detoxification from opiates in prison. Mothers 10 and 11 were identified during their first trimester and they both accepted treatment within the antenatal clinic. They were initially stabilised on a prescription of between $10 \mathrm{mg}$ to $15 \mathrm{mg}$ methadone mixture daily. During their second trimester the drug was gradually withdrawn so that they achieved abstinence by their third trimester. They remained drug-free until delivery as confirmed by urine testing, and there were no neonatal withdrawal symptoms. Neither had any previous contact with drug treatment agencies and they had been using opiates for at least four years before becoming pregnant. They had both begun to reduce their opiate consumption when they realised they were pregnant, one switching from heroin to physeptone and the other restricting herself to smoking heroin only. The 
partner of one of these patients' also underwent an out-patient opiate detoxification. This particular mother also attended a post detoxification 'non-user' group at a drug dependency unit. The final and 12th mother was identified as using heroin by way of the urine survey. Shechose to remain anonymous and was therefore not assessed. During the 12 months of the project no mothers gave birth to babies with opiate withdrawal symptoms other than those mentioned above. No miscarriages through opiate misuse were identified, although this was clearly difficult to detect.

Of the four mothers identified as using cocaine, three volunteered for assessment. One was a polydrug abuser with a long history of treatment from a drug dependency clinic. The other two had never been exposed to treatment although one admitted to smoking cocaine daily before becoming pregnant. The only identified amphetamine user was also interviewed and, despite using daily up to $\frac{1}{4} \mathrm{gm}$ amphetamine in the past, she too had never undergone treatment. The sedative and alcohol users rarely came forward and the two mothers who did flatly denied drinking alcohol, despite evidence to the contrary.

\section{Another service}

The neighbouring borough of Lambeth is served by the St Thomas's Hospital Drug Dependency Unit and its antenatal clinic treats a larger number of patients per year than its equivalent in Guy's Hospital.

The two services were compared. Because of staff changes which disrupted the St Thomas's drug dependency unit during 1988, the 12 month period from January to December 1987 was examined. Nine pregnant opiate users were identified (Onen, 1987). Six of these mothers were existing patients of the clinic who had been receiving prescribed methadone for periods ranging from one to 17 years prior to becoming pregnant. Two were referred by their GPs and both had previous contact with either drug dependency clinics or other agencies. The remaining patient was identified only at term. Although she admitted using a variety of opiates neither she nor her baby showed signs of opiate withdrawal symptoms at delivery.

Difficulties arose in the management of these patients. One of the mothers concealed her pregnancy from the clinic, while her partner, who was also a patient of the clinic, collected her prescription on her behalf. However she apparently withdrew from opiates on her own accord and at delivery her baby did not develop withdrawal symptoms. One patient returned to Dublin within the first weeks of her pregnancy. Of the remaining six mothers, all were still taking opiates at term and in only two cases did the neonates not develop withdrawal symptoms. Five of these mothers required at least one admission during their pregnancies; one mother was admitted four times. Benzodiazepine and barbiturate poly-drug abuse figured prominently in three of these mothers' admissions.

\section{Comment}

The number of mothers detected using drugs or alcohol during pregnancy was low. However, this may be an underestimate as mothers may restrict their consumption at the time of their first visit to the antenatal clinic. There was also a variation in results between the surveys, particularly for alcohol, but this may be a reflection of the small numbers involved. Although we cannot exclude prescribed drugs, the surveys showed that up to $8 \%$ of pregnant mothers had alcohol or drugs in their urine. Cannabis was the commonest substance at $6.5 \%$, then opiates at $2 \%$, followed by alcohol, stimulant and sedative drugs in that order. The alcohol tests were sensitive to a blood alcohol level of $10 \mathrm{mg} \%$ and as they were usually carried out in the morning were probably indicative of unhealthy drinking during pregnancy. Our findings on alcohol are similar to those of other studies (London \& Lipsedge, 1988). We believe these drug urine surveys to be the first of their kind to be carried out in the United Kingdom. In the United States Walberg (1986) collected urine from neonates using a specially designed bag. He found a different drug profile with cocaine the commonest drug, followed by opiates, phencyclidine third and alcohol last. He did not test for cannabis.

The number of pregnant drug users undergoing treatment in any one year is not great. Eight pregnant addicts were treated at the St Thomas's Hospital drug dependency unit while at Guy's Hospital only seven mothers underwent treatment. The majority of the former were existing patients of the drug dependency clinic who became pregnant while on longterm prescriptions of opiates. The number of cases is, however, rising, probably due to the increasing use of illicit substances in the general population. Between 1970 and 1975 Ghodse et al (1977) described five cases who maintained contact throughout their pregnancies with a drug dependency unit in the East End of London. In 1986 Riley (1987) reported 29 cases treated at University College Hospital.

In determining the extent of the problem, one is essentially dealing with two groups of drug users. There are those who on falling pregnant were already in treatment or were treated in the past. They have crossed that boundary in conceptualising their drug use as a problem requiring help. All eight patients treated by the St Thomas's Hospital drug dependency unit and four of the eight mothers assessed in Guy's Hospital had received treatment for opiate 
misuse before their pregnancies. The second group are those who have not and may never cross that threshold. When setting up the Pregnant Misuser Project the service was designed, in fashionable parlance, to be 'user-friendly'. Mothers were offered direct access to treatment with as few obstacles as possible and within the framework of the antenatal clinic. Despite this, many did not cross that conceptual boundary. A third of mothers identified as opiate users chose not to be seen, while of the four assessed and without histories of past treatment, only one underwent treatment solely within the antenatal clinic. Thus it would appear that enhancing the "userfriendliness' of services does not noticeably engage more patients in treatment. Without being able to ask these mothers, one can only speculate on the reasons. Guilt over taking drugs during pregnancy, fear of labelling, issues of legality, are some explanations that come to mind.

It is the group of mothers who have not conceptualised their drug or alcohol use as a problem who should give most cause for concern. Most of those assessed in the project, who had no history of treatment, nevertheless admitted to worrying levels of consumption at some point in their lives. These mothers may come into contact only with primary care workers or antenatal clinic staff. Although they may not view their drug use as a problem in itself, they may be prepared to re-examine it in the light of their pregnancies. It seems logical, therefore, for these health professionals to be the ones to advise and provide simple interventions; activities which midwives practise in other areas of their work. In order to achieve this, these professionals must firstly see such a role as legitimate for themselves. They must feel adequate within such a role, possessing the necessary knowledge to be able to discuss the issues with patients. Finally, they should be able to receive support and consult with specialised workers in the addiction field when necessary. This approach was first developed in the alcohol treatment field with the establishment of Community Alcohol Teams (Shaw et al, 1978).

The main problem, however, would be in persuading midwives and other health professionals to see this as a legitimate role for themselves. The pregnant mother who conceals her drug use often engenders less anxiety than the chaotic polydrug user already engaged in treatment. It is the addict who is easily identified through her appearance and behaviour who evokes the strongest feelings among the staff.

Although the number of pregnant mothers physically dependent on drugs may be small, there is a wider public health problem involving those misusing drugs and alcohol during pregnancy. Routinely screening urine to detect those mothers is not cost effective and would be impracticable. Services for pregnant drug misusers should not be seen as the preserve of specialised agencies and the role of other professionals, such as midwives and general practitioners, should be recognised, fostered and encouraged.

\section{References}

Ghodse, A. H., ReED, J. L. \& MACK, J. W. (1977) The effect of maternal narcotic addiction on the newborn infant. Psychological Medicine, 7, 667-675.

LONDON, M. \& LIPSEDGE, M. (1988) Drug and alcohol misuse in pregnancy. British Medical Journal, 297, 68-69.

ONEN, T. S. (1987) Personal communication.

RuLE, D. (1987) The management of the pregnant drug addict. Bulletin of the Royal College of Psychiatrists, 2, 362-365.

Shaw, W., Cartwright, A., Spratley, T. \& Harwin, J. (1978) Responding to Drinking Problems. London: Croom Helm.

WALBERG, C. B. (1986) Incidence of drug abuse in newborns in Los Angeles. Proceedings of the International Association of Forensic Toxicologists, 23rd International Meeting, 55-60. 